\title{
DE LA “CHACRA” A LA “OBRA". MIGRANTES PARAGUAYOS DE EXTRACCIÓN RURAL EN LA INDUSTRIA DE LA CONSTRUCCIÓN DEL ÁREA METROPOLITANA DE BUENOS AIRES
}

\author{
Going from the farm to the site. Paraguayan rural workers in the \\ construction industry of Buenos Aires
}

Álvaro Alejandro Del Águila*

\begin{abstract}
Resumen. Este trabajo pretende analizar el proceso migratorio de trabajadores paraguayos hacia la Argentina y su posterior inserción laboral en la industria de la construcción del Área Metropolitana de Buenos Aires. A través de la presentación conjunta de fragmentos de entrevistas y fuentes secundarias se pretende rastrear el origen de los trabajadores de la construcción del Área Metropolitana de Buenos Aires en las distintas localidades de Paraguay, con el objeto de discutir la posibilidad de considerar a la industria de la construcción como un "nicho laboral" particular que logra captar la fuerza de trabajo de un número importante de migrantes paraguayos de origen rural. Como producto del análisis realizado, se concluye que si bien existen importantes indicios que llevan a creerlo, no es posible establecer explicaciones de tipo lineal que relacionen la proveniencia rural de los migrantes con la inserción laboral en la industria de la construcción.
\end{abstract}

Palabras clave: industria de la construcción, Paraguay, Argentina.

\begin{abstract}
This paper aims to analyze the migration process of Paraguayan rural workers to Argentina and their subsequent employment in the construction industry of the Metropolitan Area of Buenos Aires. Through the joint presentation of fragments of interviews and secondary sources, the aim is to track the origin of workers in the construction of the Metropolitan Area of Buenos Aires in the different localities of Paraguay, in order to discuss the possibility of considering Construction as a particular "job niche" that manages to capture the workforce of a significant number of Paraguayan migrants of rural origin. As a result of the analysis carried out, it is concluded that although there are important indications that lead to believe it, it is not possible to establish linear explanations that relate the rural provenance of the migrants with the labor insertion in the construction industry.
\end{abstract}

Keywords: construction industry, Paraguay, Argentina.

\footnotetext{
* Centro de Estudios e Investigaciones Laborales (CEIL-CONICET). Buenos Aires, Argentina.
} 


\section{Introducción}

La producción académica referida a movimientos migratorios desde países limítrofes hacia la Argentina ha crecido significativamente en las últimas décadas ${ }^{1}$. Entre el vasto conjunto de producciones, sólo algunas han enfocado en el caso de los migrantes paraguayos ${ }^{2}$, siendo aún menor el número de trabajos que ha buscado recuperar las trayectorias laborales previas de este colectivo con el objeto de señalar los contrastes respecto de sus inserciones laborales posteriores en la sociedad de destino ${ }^{3}$.

A partir de esto, el presente trabajo busca contribuir a la cuestión analizando las características que adopta el proceso migratorio de varones paraguayos hacia la Argentina y su posterior inserción laboral en la industria de la construcción del Área Metropolitana de Buenos Aires (AMBA). A través de la presentación conjunta de fragmentos de entrevistas ${ }^{4}$ y fuentes secundarias se busca, por un lado, dar cuenta de las dimensiones del flujo de personas provenientes de ese país en términos de etapas y magnitudes y, por otro, presentar la mirada de los propios sujetos respecto de esos mismos procesos.

1 Cf. BALÁN, Jorge. Migraciones temporarias y mercado de trabajo rural en América Latina; BALÁN, Jorge. La economía doméstica y las diferencias entre los sexos en las migraciones internacionales: un estudio sobre el caso de los bolivianos en la Argentina; BENENCIA, Roberto. Apéndice: La inmigración limítrofe; BENENCIA, Roberto, KARASIK, Gabriela. Inmigración limítrofe: los bolivianos en Buenos Aires; CERRUTTI, Marcela. Diagnóstico de las poblaciones de inmigrantes en la Argentina; DEVOTO, Fernando. Historia de la inmigración en la Argentina; GRIMSON, Alejandro. Fronteras, Estados e identificaciones en el Cono Sur; GRIMSON, Alejandro. Nuevas xenofobias, nuevas políticas étnicas en Argentina; MAGUID, Alicia. Migrantes limítrofes en el mercado de trabajo del área metropolitana de Buenos Aires; MARSHALL, Adriana, ORLANSKY, Dora. Inmigración de países limítrofes y demanda de mano de obra en la Argentina, 1940 1980; por nombrar sólo a algunos.

2 Cf. BRUNO, Matías. Las experiencias laborales de paraguayos en Buenos Aires. Un análisis de movilidad ocupacional inicial; BRUNO, Sebastián. Movilidad Territorial y laboral de los migrantes paraguayos en el Gran Buenos Aires; BRUNO, Sebastián. Inserción laboral de los migrantes paraguayos en Buenos Aires. Una revisión de categorías: desde el "nicho laboral" a la "plusvalía étnica"; CERRUTTI, Marcela, BRUNO, Matías. La inserción de migrantes paraguayos y peruanos en el mercado de trabajo del Área Metropolitana de Buenos Aires; COURTIS, Corina, PACCECA, María Inés. Género y trayectoria migratoria: mujeres migrantes y trabajo doméstico en el Área Metropolitana de Buenos Aires; DEL ÁGUILA, Álvaro. A través de la yerba: etnicidad y racionalidad económica entre los trabajadores rurales paraguayos en la industria de la construcción de Buenos Aires; MIRANDA, Ana (comp.). Ahata Che: Juventud, migración y género en el corredor Paraguayo-Argentino.

3 BRUNO, Matías, op. cit.; CERRUTTI, Marcela, PARRADO, Emilio. Migración laboral de trabajadores paraguayos a la Argentina: entrada a los mercados de trabajo y trayectorias ocupacionales; CRAGNOLINO, Elsa. Género, trabajo y familia. Trayectorias laborales de mujeres de origen campesino en el norte de Córdoba, Argentina; MIRANDA, op. cit.

4 Las entrevistas que se presentan fueron realizadas entre 2006 y 2015 en distintas obras en construcción del AMBA. Por cuestiones de espacio, no podremos detenernos en un análisis pormenorizado de las vicisitudes y particularidades del proceso de trabajo etnográfico. Un análisis detallado del abordaje metodológico que sustenta la investigación puede verse en Del Águila, op. cit. 
Una vez hecho esto, se intenta rastrear en las estadísticas e historias de vida el origen de los trabajadores de la construcción del AMBA en las distintas localidades de Paraguay, con el objeto de discutir la posibilidad (o no) de considerar a la industria de la construcción como un "nicho laboral" particular que logra captar la fuerza de trabajo de un número importante de migrantes paraguayos de origen rural en el AMBA.

Si bien en otras oportunidades abordamos la presencia de trabajadores paraguayos de extracción rural en las obras del AMBA atendiendo a sus principales dimensiones y manifestaciones socioculturales ${ }^{5}$, no nos fue posible en ese momento referir a los procesos más amplios que estructuran dicho fenómeno. Por el contrario, en aquella oportunidad nos limitamos a analizar en detalle el proceso de adaptación de estos hombres a la lógica productiva de la industria capitalista, interpretando dicho proceso en términos de "proletarización étnica" ${ }^{1}$. A través de este concepto, pretendimos dar cuenta de la "metamorfosis" que experimentaban los sujetos (de pequeños productores orientados a la subsistencia a obreros que perciben un salario regular a cambio de la venta de su fuerza de trabajo). Dicha proletarización adquiría características particulares, en la medida en que no se trataba de una mera migración interna del tipo campo-ciudad, sino que implicaba el atravesamiento de los límites nacionales, dando lugar a la adquisición no proyectada de un oficio (el de "constructor") por parte de los migrantes. Mostramos así que la inserción de un número significativo de migrantes paraguayos de origen rural en las obras del AMBA no parecía responder a ningún tipo de pericia o idoneidad por parte de éstos, y que por el contrario se vinculaba a procesos de índole sociocultural a través de los cuales los empleadores argentinos interpretaban a estos migrantes como seres "naturalmente más aptos para el trabajo duro". Así, a través de una serie de mecanismos no-formales (como las relaciones sociales basadas en el parentesco, la vecindad o el paisanaje), el empresariado argentino de la construcción accedía a un flujo de fuerza de trabajo no proletarizada. De este modo, y por ocurrir en contextos caracterizados por la hipervisibilización de la diferencia étnica y nacional, la inserción laboral de estos migrantes daba lugar a experiencias laborales más precarizadas que las de otros trabajadores.

Dicho esto, en esta oportunidad nos abocaremos a abordar la cuestión desde otra arista, buscando ahora rastrear en las estadísticas e historias de vida el origen de los trabajadores de la construcción del AMBA en las distintas localidades de Paraguay, con el objeto de determinar hasta qué punto es posible pensar a la industria de la construcción del AMBA como un escenario laboral destacado para los migrantes paraguayos de origen rural.

\footnotetext{
5 Ibidem.

6 Ibidem.
} 
El artículo se compone de cuatro partes. En la primera, se reconstruye a partir de datos estadísticos y fuentes secundarias la presencia de los migrantes paraguayos en el AMBA. A continuación, se dedica una segunda sección a caracterizar a través de relatos y fuentes secundarias la presencia específica de varones paraguayos en las obras en construcción del AMBA. Una vez hecho esto, se rastrean los departamentos de origen de los trabajadores que se desempeñan en las obras, con el objeto de intentar esclarecer las relaciones que parecen existir entre la marcada extracción rural de una gran parte de ellos y el trabajo en la construcción en el AMBA. Por último, se esbozan algunas reflexiones respecto de lo analizado, con el objeto de sopesar el camino realizado y dejar abiertos nuevos interrogantes hacia el futuro.

\section{Los migrantes paraguayos en el Área Metropolitana de Buenos Aires}

Distintos autores ${ }^{7}$ han coincidido en señalar que, en un primer momento, los migrantes paraguayos habrían respondido a las demandas coyunturales de mano de obra o a trabajos temporarios en cosechas, principalmente en las áreas limítrofes o fronterizas. Para Maguid ${ }^{8}$, un segundo momento se habría iniciado cuando los migrantes comienzan a cubrir, a partir de 1947, los puestos de trabajo en las industrias regionales y mineras que empiezan a ser abandonados por los migrantes internos que dejan sus provincias natales para trasladarse hacia la capital y el Gran Buenos Aires.

\section{Cuadro 1 - Nacidos en el Paraguay residentes en la Argentina según lugar de residencia 1895-2010}

\begin{tabular}{c|c|c|c}
\hline Año & $\begin{array}{c}\text { Residentes Paraguayos en } \\
\text { Argentina }\end{array}$ & $\begin{array}{c}\text { Porcentaje de Paraguayos } \\
\text { residentes en AMBA }\end{array}$ & $\begin{array}{c}\text { Porcentaje de paraguayos } \\
\text { residentes en otras provincias }\end{array}$ \\
\hline 1869 & 3.288 & Sin datos & Sin datos \\
\hline 1895 & 14.562 & $9,30 \%$ & $90,70 \%$ \\
\hline 1914 & 28.049 & $11,20 \%$ & $88,80 \%$ \\
\hline 1917 & 93.248 & $12,40 \%$ & $87,60 \%$ \\
\hline 1947 & 155.269 & $13,30 \%$ & $86,70 \%$ \\
\hline 1960 & 230.000 & $29,60 \%$ & $70,40 \%$ \\
\hline 1995 & 250.450 & $73,30 \%$ & $26,70 \%$ \\
\hline 2010 & 550.713 & $75,40 \%$ & $24,60 \%$ \\
\hline
\end{tabular}

Fuente: elaboración del autor en base a datos consignados en FISCHER, Sara, PALAU, Tomás, PEREZ, Noemia. Inmigración y emigración en el Paraguay 1870 - 1960; PASTORE, Carlos. La lucha por la tierra en el Paraguay; FLORES COLOMBINO, Andrés. La fuga de los intelectuales. Emigración paraguaya; CARRÓN, Juan María. Shifting patterns in Migration from bordering countries to Argentina: 1914-1970; Indec. Censo de Población, Hogares y Viviendas 2010.

BENENCIA, KARASIK, op. cit.; MARSHALL, ORLANSKY, op. cit.; BALÁN, La economía doméstica..., op. cit.; MAGUID, op. cit.

8 Ibidem. 
A partir de 1960, con la caída de los precios de los productos regionales y la incorporación de tecnología de mecanización de la cosecha en el noroeste, la migración paraguaya (y la limítrofe en general) habría cambiado de rumbo, buscando nuevas oportunidades en el AMBA, en los rubros de la construcción, la industria manufacturera y el servicio doméstico, por estar estas tareas mejor remuneradas que en el resto de las provincias argentinas o en sus propios países ${ }^{9}$.

\section{Cuadro 2 - Extranjeros y extranjeros limítrofes por año censal}

\begin{tabular}{c|c|c|c}
\hline Censo & $\begin{array}{c}\text { \% de extranjeros sobre } \\
\text { población total }\end{array}$ & $\begin{array}{c}\text { \% de limítrofes sobre } \\
\text { población total }\end{array}$ & $\begin{array}{c}\text { \% de limítrofes sobre } \\
\text { extranjeros }\end{array}$ \\
\hline 1869 & 12,1 & 2,4 & 19,7 \\
\hline 1895 & 25,4 & 2,9 & 11,5 \\
\hline 1914 & 29,9 & 2,6 & 8,6 \\
\hline 1947 & 15,3 & 2 & 12,9 \\
\hline 1960 & 13 & 2,3 & 17,9 \\
\hline 1970 & 9,5 & 2,3 & 24,2 \\
\hline 1980 & 6,8 & 2,7 & 39,6 \\
\hline 1991 & 5 & 2,5 & 50,2 \\
\hline 2001 & 4,2 & 2,6 & 60,3 \\
\hline 2010 & 4,5 & 3,1 & 68,9 \\
\hline
\end{tabular}

Fuente: elaboración del autor a partir de datos presentados en Maguid (op. cit.), Cerrutti (op. cit.) y completada con el análisis de censos nacionales argentinos (1980, 1991, 2001, 2010).

En relación a esta creciente "metropolización" de la migración paraguaya, trabajos recientes ${ }^{10}$ han mostrado que el patrón de residencialidad de los migrantes se ha visto modificados en las últimas décadas, cuando estos comienzan a dirigirse de forma directa hacia las grandes ciudades del país, en general, sin hacer escalas previas en otras provincias de la Argentina. Así, durante la década de 1990, de acuerdo a Maguid ${ }^{11}$, el AMBA se consolidaría como destino principal de paraguayos y bolivianos $(65 \%$ y $39 \%$ del total de residentes en el país, respectivamente).

Las prioridades son la gente que está llegando en esta nueva etapa de la migración paraguaya... son gente joven que vienen ya en familia, no como los paraguayos de antes que venían o papá o mamá, veía qué encontraba, después iba sumando a la familia... ahora vienen todos, vienen de a tres, de a cuatro $y \ldots$ sin ninguna infraestructura... no tienen casa, no tienen trabajo asegurado, no tienen nada... ni documentos, ni nada (Entrevista

\footnotetext{
9 BALÁN, La economía doméstica..., op. cit.; MAGUID, op. cit.

${ }^{10}$ BRUNO, Inserción laboral..., op. cit.

11 Ibidem.
} 
a Andrés. Trabajador de la construcción y miembro de una organización social paraguaya de José C. Paz. Febrero de 2011).

Una cuestión de interés se vincula al hecho de que, a pesar de los vaivenes económicos argentinos, desde mediados de la década de 1970 la migración proveniente de países limítrofes (y de Paraguay en particular) no solo no mermó sino que continuó aumentando ${ }^{12}$.

Hoy en día en La Plata, casi el 80\% de todos los paraguayos que viven... y por ahí nos podemos quedar cortos... viven en terrenos usurpados... y definitivamente, prácticamente hoy en día, más de 25 o 30 años que están viviendo acá... nosotros en este momento estamos avanzando bastante fuerte con la Provincia, que se pueda sacar una ley, o un decreto... que por única vez se puedan escriturar todos esos terrenos, a través de la ley Pierri, a través de Usucapión...y estamos esperando, de un momento a otro que la Provincia, junto con el Embajador, firmen ese convenio (Entrevista a Sinforiano, antiguo trabajador de la construcción y actual miembro de una organización social paraguaya de José C. Paz. Febrero de 2011).

Hacia fines de los '90, Cacopardo y López ${ }^{13}$ estimaban que el 53,2 \% de los paraguayos residentes en Argentina lo hacía en el conurbano bonaerense, puntualmente en dos zonas. La primera comprendía los partidos de La Matanza, Esteban Echeverría, Almirante Brown y Quilmes, mientras que la segunda estaba integrada por los partidos de General Sarmiento, Moreno y Morón. Además, las autoras estimaron que un 20\% de las familias paraguayas radicadas en el país por esos años residían en La Matanza.

Porque el asentamiento que tenemos nosotros que acá, cuando empezamos a trabajar con el relevamiento, tiene una problemática triple, a corto plazo que es la siguiente: tiene propietario el terreno. Era una olería que, bueno, no pudo pagar sus impuestos al Municipio, que la hizo cerrar, quedó ahí, los espacios vacíos que fueron ocupados... incluso, en una parte se hicieron las casita, con los planes y demás... lo que quedó, ocuparon ahora los paraguayos, se llama 'el barrio de los paraguayos' lo que es fundamental entre nosotros es que a raíz de esto que pasó... de la muerte de un compatriota en el Indoamericano... que se insista a la gente que la usurpación no es buena... que no es buena para nada... entonces tiene que tener conocimiento de que está en país extranjero, y que tiene que luchar para conseguir, como hicieron todos los que vinieron anteriormente... para brindar, para brindar algo mejor a la familia... Entonces, a partir de ahí, es otro asesoramiento más... y que eso se puede lograr de otra manera... de formas legales... por ejemplo lo que pasó con las unificaciones con el Municipio... entonces, el Municipio

\footnotetext{
12 Ibidem.

${ }^{13}$ CACOPARDO, María, LÓPEZ, Silvia. Familia, trabajo y fecundidad de los migrantes de países limítrofes.
} 
puede ayudar mucho en ese sentido... construyendo política (Entrevista a Sinforiano, José C. Paz. Febrero de 2011).

De acuerdo a datos más recientes, el porcentaje de extranjeros en el Gran Buenos Aires representa un 7,5\% sobre la población total ${ }^{14}$. En cuanto al análisis por partidos, aquellos que poseen las mayores proporciones de población nacida en el extranjero con respecto a la población total son: Esteban Echeverría (10,1\%), La Matanza (9,7\%) y Lomas de Zamora (9,3\%). De estos extranjeros, se registra una importante presencia de personas nacidas en el Paraguay, las que representan un 58,6\% del total de los no nativos. Le siguen en orden de importancia los oriundos de Bolivia que representan el $18,2 \%{ }^{15}$.

\section{Los varones paraguayos y las obras en construcción en Buenos Aires}

Con respecto a la inserción laboral de migrantes limítrofes en la industria de la construcción, varios autores coincidieron en señalar que el rápido y continuo desarrollo de la construcción residencial privada a partir de 1960, empleó grandes cantidades de mano de obra adicional en el corto plazo, sobre todo paraguayos y bolivianos que se fueron sumando a los migrantes internos y nativos del área que se estaban reubicando en esta rama a causa de su carácter dinámico y expansivo ${ }^{16}$.

Una reflexión que merece destacarse es aquella que refiere al modo en que los propios migrantes han interpretado las distintas "oleadas" migratorias de sus connacionales. Al menos discursivamente, muchos entrevistados distinguieron entre distintas "calidades" de migrantes.

Por ahí no sé si es lo mismo, porque nosotros estamos hablando por nosotros, pero hay gente, en otro lado, por ejemplo en La Matanza, la gente nueva que vinieron... yo no creo que les resulte tan fácil... acomodarse... primero, porque no tienen escala, vienen del campo a una gran ciudad que le es desconocido... por ahí, nosotros... no sé, pero... al venir de joven, te integras más rápido... depende las épocas también, no? Pero, por ejemplo, ellos los que vienen ahora, vienen sin escala, ni siquiera en Asunción... no pasan... pero mucha gente...vos ves gente, con muchas necesidades... porque allá no hay trabajo todavía... se está armando Paraguay con su nuevo presidente... entonces cuesta, este período de transición es bravo... (..) hay muchos paraguayos que vienen por salud que acá la Argentina no pregunta de dónde venís ni quién sos, ni si tenés documentos... la Argentina te abre los hospitales, con una solidaridad que nosotros... por eso somos tan agradecidos... la salud

\footnotetext{
${ }^{14}$ INDEC. Microdatos por trimestres de la Encuesta Permanente de Hogares. Período 2004-2013.

15 Ibidem.

${ }^{16}$ BENENCIA, KARASIK, op. cit.; MARSHALL, ORLANSKY, op. cit. De acuerdo a procesamientos propios realizados sobre el Censo de Población, Hogares y Viviendas 2010 (Indec), de 206.538 personas de nacionalidad paraguaya censadas en el Gran Buenos Aires, cerca de 56.008 se desempeñaban en ocupaciones vinculadas a construcción e infraestructura.
} 
en Paraguay es muy precaria... por eso vienen aquí a... lo único que... la documentación de los argen... de los paraguayos, tendría que ser un poco más estricta, no? Habría que apretar un poquito más ese tema... si, si... yo por ejemplo, no estoy para nada de acuerdo con la gente que quiere aprovechar... y que lo han hecho... a mí me pone mal... porque, también hay gente de acá... claro... hay gente de acá, por ejemplo... claro, está bien... la gente ya no quiere trabajar... la gente quiere planes sociales... el paraguayo, por lo general, no viene a pedir eso porque le es difícil, si no tiene documentos, no tiene tantos años... no pide eso pero pide trabajo... bueno, el trabajo lo consigue y, después, por ahí, el argentino que viene de afuera también le pasa lo mismo, ya no quiere ir a trabajar en la obra... como hacía antes, por ejemplo, el paraguayo... el trabajo que se descartaba era lo que agarraba... y ahora, por ejemplo, ahora... yo he escuchado "estos paraguayos de mierda" que vienen y te sacan el trabajo... pero, esas cosas... el paraguayo viene a trabajar... yo no sé si ahora vienen con otras ideas... pero yo, en la cabeza creo que pasa lo mismo acá con la gente más joven... el paraguayo... el migrante viejo, es muy agradecido... y siempre está diciendo "Argentina me dio mucho"... aparte de los hijos y todas las otras cosas... entonces, de por sí, está agradecido... entonces, si la gente es así, el que convive, y es de acá, tiende a no discriminarlo... pero el que viene de allá y que se trae puesto el sombrero y... un montón de cosas... si, es lógico que alguien lo discrimine... porque de repente no cumple todas las reglas... el tema del documento es algo muy importante... yo creo que es un poquito de irresponsabilidad... de dejadez... porque... cuando tiene tiempo, no lo hace y después, no tiene tiempo y... el documento lleva su proceso... aunque ahora es mucho más rápido, eh... no hay excusas, ahora no hay excusas... es rapidísimo y te lo mandan a tu casa... solamente hay que tomarse el tiempo... porque acá hay paraguayos que tienen hijos argentinos y aún no tienen el documento... claro... y las instituciones argentinas deberían apretar un poquito más... a mí me parece que eso es algo fundamental porque el paraguayo... se aboca a trabajar... a trabajar...de lunes a sábado a trabajar... total, no me exigen" (Entrevista a Blanca e Isabel, miembros de una organización social paraguaya de Berazategui. Enero de 2011).

En términos generales, y más allá del año de proveniencia, si desagregamos las ocupaciones que realizan los varones paraguayos podremos ver aquello que señalaba Bruno ${ }^{17}$ y que se vincula a que cuatro de cada diez varones paraguayos en el AMBA trabaja en la construcción:

${ }^{17}$ BRUNO, Movilidad Territorial..., op. cit. 


\section{Cuadro 3 - Varones no migrantes y migrantes paraguayos residentes en Gran Buenos Aires ocupados según grupos ocupacionales. \\ Primer semestre 2003 (\%)}

\begin{tabular}{c|c|c}
\hline Grupos ocupacionales & No migrantes (Argentinos) & Migrantes paraguayos \\
\hline Total & 100 & 100 \\
\hline Prestación de servicios & 1.870 .381 & 67.535 \\
\hline Construcción & 73,8 & 35,1 \\
\hline Producción de bienes no agropecuarios & 10,4 & 39,8 \\
\hline Producción de bienes agropecuarios & 15 & 23,8 \\
\hline Servicio doméstico & $0,5^{* *}$ & $0,8^{* * *}$ \\
\hline Sin información & $0,1^{* *}$ & $0,6 * * *$ \\
\hline
\end{tabular}

Nota: EPH: ** CV mayor a $20 \%$. ECMI: *** CV mayor a 25 \%. Fuente: Procesamientos de Sebastián Bruno en base a EPH (onda mayo 2003) y ECMI (BRUNO, Sebastián, DEL ÁGUILA, Álvaro. Huellas de tierra roja en el cemento porteño. Trabajadores migrantes paraguayos de la construcción en Buenos Aires).

Un dato que destaca es el que muestra que los migrantes paraguayos en Argentina no se han insertado de forma significativa en tareas agrícolas $(0,8 \%)$. Por el contrario, la prestación de servicios y la construcción parecen representar las inserciones más relevantes. Nos enfrentamos aquí al objeto principal de este trabajo, y que se vincula al intento de responder al siguiente interrogante: ¿Es posible hablar de una mayor presencia de trabajadores paraguayos de "extracción rural" en las obras de construcción del AMBA en comparación a otros sectores? Para averiguarlo, tendremos que indagar respecto de en qué parte de Paraguay vivían los migrantes antes de llegar a Argentina.

\section{Los departamentos de origen de los trabajadores paraguayos de la construcción del Área Metropolitana de Buenos Aires}

La región de origen de los actuales trabajadores de la construcción constituye uno de los datos más ricos para caracterizar las trayectorias laborales, ya que permite estimar hasta cierto punto el grado de experiencia previa antes de emigrar. En un trabajo anterior ${ }^{18}$, notamos que quienes venían desempeñándose en actividades rurales en Paraguay terminaron absorbidos en gran medida por la construcción del AMBA, siendo que un tercio de la fuerza de trabajo "constructora" paraguaya en Buenos Aires provenía del "campo". Al mismo tiempo, en otra oportunidad afirmamos que este gran grupo de trabajadores no experimenta un mero cambio de actividad al llegar a Buenos Aires, sino que también debe insertarse en nuevas relaciones de trabajo y producción, lo que acarrea importantes transformaciones de orden simbólico ${ }^{19}$.

\footnotetext{
18 BRUNO, DEL ÁGUILA, op. cit.

19 DEL ÁGUILA, op. cit.
} 


\begin{abstract}
"Lezcano"
Lezcano tiene 62 años y es oriundo de Itá, localidad situada a $35 \mathrm{~km}$. de Asunción. Vino a los 22 años a Argentina, en 1968. Una vez llegado, se estableció en Villa Cildañez, en el barrio porteño de Parque Avellaneda. Desde mediados de la década de 1980, vive junto a su familia en Laferrere, partido de La Matanza, provincia de Buenos Aires. Se define a sí mismo como "constructor en general". En Itá, Lezcano vivía con su madre. Él fue su único hijo. Vivían en una granjaque su madre había establecido en un pedazo de tierra desocupada. Ella se dedicaba allí a las actividades propias de una granja: era matarife de chanchos y vendía gallinas y huevos. Solo para uso doméstico, plantaban en un pequeño terreno algo de caña y de mandioca. No conoció a su padre hasta avanzada edad. Años más tarde, también descubrió que tenía varios hermanos por parte de él. Mantiene relación con algunos de ellos en la actualidad. Su padre se desempeñaba como hachero en algunos campos de la zona (Nota de campo a partir de conversación con "Lezcano". Febrero de 2008).
\end{abstract}

Como podemos ver, Lezcano vivía en Itá, una ciudad importante cercana a Asunción. Sin embargo, antes de emigrar, sus labores se enmarcaban fundamentalmente en la producción de granja. Si bien es sabido que la proveniencia geográfica de los migrantes en general es sumamente heterogénea ${ }^{20}$, es interesante observar particularmente la información desagregada para el caso de los trabajadores de la construcción:

\title{
Cuadro 4 - Migrantes paraguayos de 18 años y más según departamento de residencia por ocupación actual. Gran Buenos Aires. Año 2003
}

\begin{tabular}{c|c|c|c|c|c}
\hline \multicolumn{3}{c|}{ Trabajadores de la construcción } & \multicolumn{3}{c}{ Resto de migrantes paraguayos } \\
\hline № Orden & Departamento & $\%$ & No Orden & Departamento & $\%$ \\
\hline & Total & 100 & & Total & 100 \\
\hline \multirow{2}{*}{1} & Itapúa & 26.800 & & & 61.958 \\
\hline 2 & Área Metropolitana de & 21,9 & 2 & Área Metropolitana de & 28,6 \\
\hline 3 & Asunción & 10,1 & 3 & Isunción & Itapúa \\
\hline 4 & Caazapá & 8,5 & 4 & Neembuacún & 12,9 \\
\hline 5 & Misiones & 5,5 & 5 & Central & 12 \\
\hline 6 & Central & 5,1 & 6 & Cordillera & 7,1 \\
\hline 7 & Cordillera & 4,9 & 7 & Caaguazú & 6,7 \\
\hline 8 & Caaguazú & 3,9 & 8 & Guairá & 5,6 \\
\hline 9 & Paraguarí & 3,4 & 9 & Misiones & 4,7 \\
\hline
\end{tabular}

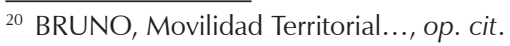




\begin{tabular}{c|c|c|c|c|c}
\hline 10 & Ñeembucú & $2,9(a)$ & 10 & Caazapá & 3,7 \\
\hline 11 & Alto Paraná & $2(a)$ & 11 & Concepción & 2,1 \\
\hline 12 & Concepción & $1,5(a)$ & 12 & Alto Paraná & 2,1 \\
\hline 13 & Amambay & $0,4(a)$ & 13 & San Pedro & $1(\mathrm{a})$ \\
\hline 14 & San Pedro & $0,3(\mathrm{a})$ & 14 & Boquerón & $0,1(\mathrm{a})$ \\
\hline 15 & Boquerón & - & 15 & Amambay & $0(\mathrm{a})$ \\
\hline & Sin información & $1,4(\mathrm{a})$ & & Sin información & 1,8 \\
\hline
\end{tabular}

Nota: a) Coeficiente de variación mayor a 25 \%. Fuente: Elaboración de Sebastián Bruno con base en ECMI 2002-2003 (BRUNO, DEL ÁGUILA, op. cit.).

Como puede verse, de acuerdo a los procesamientos realizados por Bruno $^{21}$, si se contrasta con el resto de los migrantes, los provenientes del Departamento de Itapúa representan más de un cuarto de la fuerza de trabajo paraguaya en la construcción argentina, "lo que sugiere la existencia de redes específicas de contactos y reclutamiento allí y de funcionamiento de la incorporación de trabajadores a través del 'paisanaje' como garantía de confianza, una vez en Buenos Aires"22.

El segundo territorio de proveniencia es el Área metropolitana de Asunción, con una proporción ligeramente menor que el resto del universo. La importancia de esa proveniencia radica en su carácter eminentemente urbano lo cual, como refiriéramos, limita las interpretaciones lineales que relacionan la construcción con un origen exclusivamente rural ${ }^{23}$.

Los departamentos de Caazapá y Misiones marcan una presencia relativa importante entre los "constructores", agrupando a casi dos de cada diez trabajadores. Dichos orígenes tienen una importancia mucho menor en el resto "no constructor", ubicados entre los departamentos menos representados respecto a la proveniencia de los migrantes ${ }^{24}$.

Sin embargo, para interpretar cabalmente estos datos, puede resultar de interés contrastar los flujos hacia la Argentina con aquellos que se dirigen a otros destinos. En este sentido, de acuerdo al estudio Ampliando Horizontes realizado por PNUD en 2008, el Departamento Central es el que registra el mayor flujo de emigrantes a España: 48,88\%. Es decir que casi la mitad de quienes emigran desde estas zonas tiene como destino ese país europeo, lo que evidencia una clara preferencia migratoria por ese destino entre las personas provenientes de las zonas más pobladas y urbanizadas del país. El mismo estudio muestra que, en contraste, los migrantes de los departamentos más empobrecidos y de población rural, como San Pedro o Caaguazú, se dirigen principalmente a

\footnotetext{
${ }^{21}$ BRUNO, DEL ÁGUILA, op. cit.

22 Ibidem, p. 8.

${ }^{23}$ DEL ÁGUILA, op. cit.

${ }^{24}$ BRUNO, DEL ÁGUILA, op. cit., p. 8.
} 
la Argentina, en un $71,5 \%$ y $72.6 \%$ respectivamente, mientras que apenas un $19,8 \%$ de las personas migrantes de San Pedro, y un 18,9\% de Caaguazú, tiene como destino España.

Por otra parte, según datos arrojados por la Encuesta Permanente de Hogares de Paraguay, hacia 2008 el número de hogares con emigrantes en estas zonas llegaba a 182.401, lo que demuestra que el porcentaje de hogares con población emigrante en el departamento de Caaguazú e Itapúa es cerca del doble que el de Asunción, San Pedro y Alto Paraná.

\section{"Cabezón"}

La experiencia de "Cabezón" o "Duhalde", como le dicen sus compañeros de trabajo, ayuda a ver otro caso concreto de migración proveniente del departamento de Itapúa. Él es originario de la localidad de Ayolas, lugar en el que fue emplazada la Represa de Yacyretá. Con 46 años, nos comentó que a su familia se le pagó un dinero al iniciarse las obras, para que se mudaran de Ayolas hacia la localidad de San Patricio, por cuestiones vinculadas a la necesidad de utilizar esos terrenos para la construcción. Una vez llegado a Buenos Aires, se instaló en José C. Paz y, a los dos meses de estar ahí, ya comenzó a trabajar en la construcción como pintor de obra, por el "contacto de un conocido" (Nota de campo sobre conversación sostenida con "Cabezón". Obra de Juana Manso y Peñaloza. Abril 2010).

El siguiente cuadro permite ver la importancia de las labores agropecuarias entre los varones paraguayos que actualmente residen en el AMBA. Puede así verse que uno de cada tres trabajadores actuales de la construcción en Buenos Aires se desempeñaba en faenas agrícolas antes de migrar.

\section{Cuadro 5 - Migrantes paraguayos de 18 años y más ocupados según última ocupación en Paraguay por ocupación actual. Gran Buenos Aires. Año 2003 (\%)}

\begin{tabular}{l|c|c}
\hline \multirow{2}{*}{ Ultima ocupación en Paraguay } & \multicolumn{2}{|c}{ Ocupación Actual } \\
\cline { 2 - 3 } & Construcción & Resto ocupados \\
\hline \multirow{2}{*}{ Total } & 100 & 100 \\
\hline Producción de bienes agropecuarios & -26.800 & -38.907 \\
\hline Inactivo en Paraguay & 34 & 18,2 \\
\hline Prestación de servicios & 20,9 & 37,3 \\
\hline Construcción & 17,4 & 19,5 \\
\hline Producción de bienes no agropecuarios & 14,5 & 9,3 \\
\hline Servicio doméstico & 12 & 14,6 \\
\hline Sin información & - & 0,1 (a) \\
\hline
\end{tabular}

Nota: (a) Coeficiente de variación mayor a 25 \%. Fuente: Elaboración de Sebastián Bruno con base en ECMI 2002-2003 (BRUNO, DEL ÁGUILA, op. cit.). 
Como puede observarse, la construcción capta más fuerza de trabajo agrícola (34\%) que, en su conjunto, el resto de los sectores productivos en Buenos Aires (18,2\%). Al mismo tiempo, estos procesamientos también permiten ver que solo algo más de uno de cada diez trabajadores actualmente ocupado en las obras se desempeñaba en la construcción en Paraguay.

Sin duda, estos datos se condicen con lo observado por nosotros durante el trabajo de campo. Así, no pudimos registrar muchos casos de actuales obreros de la construcción que ya se hubieran desempeñado como tales antes de migrar. Es puntualmente a partir de esta situación que argumentaremos que una parte importante de los trabajadores rurales paraguayos se proletariza/ asalariza por vez primera en la construcción del AMBA (uno de cada tres migrantes hoy en las obras trabajaba en la producción agrícola). Sin embargo, por supuesto, esta interpretación posee ciertas limitaciones.

\begin{abstract}
"Esquivel"
Tiene 37 años, nació en Asunción. Su padre tenía allí una tapicería y se dedicaba principalmente a la confección de los asientos para las líneas de colectivos. Desde los once años, Esquivel ayudó en el negocio familiar y, desde los catorce, cuando empieza la secundaria, ya se "bancó solo" con su trabajo. Concluyó la educación primaria y secundaria y realizó los primeros dos años de una carrera terciaria en Economía. Incluimos aquí su caso porque permite relativizar algunos planteos relacionados a la supuesta movilidad social ascendente perseguida por los migrantes al arribar a Buenos Aires. En el caso de Esquivel, la construcción no es el primer rubro en el cual se inserta una vez en Buenos Aires. Por el contrario, abre una tapicería en Moreno, en la casa de su hermano mayor, que "hace veinte años está acá". En relación al motivo de su venida a Buenos Aires, dijo que en Paraguay "La tapicería no iba más...aparte... yo ya me había juntado... y yo tenía mi tallercito... con mi hermano... después él se fue para Brasil, no Brasil, Paraguay, pero del lado de Brasil... se puso su taller... yo me quedé en Asunción... yo me junté... y bue... por esas cosas de la vida... bueno... se armó quilombo con mi mujer... y decidí venirme (Entrevista a "Esquivel". Obra de la calle Thorne. Febrero 2008).
\end{abstract}

La entrevista a Esquivel nos permite visualizar algunas cuestiones interesantes. En primer lugar, él proviene de Asunción. Recordemos que solo uno de cada cinco obreros paraguayos en las obras del AMBA proviene de la capital del país $(21,9 \%)$. Según nos cuenta, él poseía un oficio previo a la emigración. Esto parece relacionarse con algo que nos comentara un contratista, aquello por lo cual los paraguayos provenientes de Asunción no se insertaron tanto en las obras como en otros rubros (zapatería, curtiembre, tapicería, etc.). Tal vez esta situación pueda relacionarse con cierta característica de los capitalinos que haga más recurrente la posesión de un "oficio". No estamos en condiciones de afirmarlo. Lo que sí nos muestra Esquivel es que, siendo 
tapicero y conociendo el rubro, lo primero que hace al llegar es establecer una tapicería. Solo una vez que esto no le resulta, acudirá a las redes sociales para conseguir un trabajo en las obras. Es decir, tanto en su caso como en el de otros como migrantes provenientes de Asunción, la construcción no representa la alternativa laboral "ideal" pero, a pesar de ello, allí terminan insertándose.

La ventaja que tienen es que vienen jugados... vienen para hacer cualquier cosa, están dispuestos a cualquier cosa... no... trabajo... vos sabés que cuando uno sale de su casa... si tiene que lavar copas, lava copas... si tiene que lavar baños, lava baños... y están... por eso... tienen esa ventaja de adaptación que no le tienen miedo a la... vos fijate, yo que estoy en el rubro de la construcción, están tres meses como ayudantes y al cuarto mes, ya te dice que es oficial, ya aprendió algo... ya se lanza con eso (Entrevista a Herminio, trabajador de la construcción. Obra de Pedro Goyena. Agosto de 2009).

En prácticamente todos los casos, los trabajadores paraguayos empiezan como "ayudantes" en la industria de la construcción argentina, permaneciendo algún tiempo en dicho escalafón hasta ascender al de "oficial".

"Darío"

Nací en Itatí, Corrientes. Y a los meses me fui a Paraguay. Nací en Corrientes porque en esa época Paraguay no tenía hospitales, entonces cuando una mujer va a tener hijos viene... esa es la realidad. Nací en Corrientes y después volví a Paraguay. Bueno, años después volví a Corrientes y empecé en la obra. Yo volví a Corrientes y ahí trabajé en la construcción. No tenía familiares, pero como me anotaron ahí me fui para ahí. Ya cuando naciste acá, te anotan como argentino. La construcción, la única más fácil que había. Tenía 20, 21 años... me vine porque allá no había nada, entonces ¿qué tenés que hacer? Tenés que venir... no había nada de laburo. Yo me vine laburando con otro muchacho, laburando ya... y me vine acá y busqué en la construcción, era lo más fácil que había... para entrar a trabajar...

A Buenos Aires llegué el 22 de Diciembre de 1975, me vine a casa de amigos, conocidos de Paraguay que estaban laburando acá en Buenos Aires y era en la obra... y me acomodaron acá... arranqué así y... me dediqué a laburar, laburar y... seguimos laburando. Laburé en muy pocas empresas eh, muy pocas... habrá cuatro empresas... hasta ahora eh, empresas constructoras... con esta, laburé 13, 14 años... entré como oficial y ahora bueno, como encargado.... Conozco todo de punta a punta, no hay problema... fui ascendiendo en la categoría... y yo estuve en otra empresa, una que se llamaba XXX, otros trece años... pero en esta entré como oficial, y así fue y ahora estoy acá... (Entrevista a Darío, Obra de 3 de Febrero y Deheza. Julio de 2014).

La entrevista a Darío muestra una serie de cuestiones de gran importancia. Él tiene 56 años y es "encargado" de la obra. Si bien nació en Corrientes, se crio en Humaitá, una localidad cercana a Pilar, en el Departamento de 
Ñeembucú. En Paraguay solo le queda un hermano, que todavía trabaja en el campo familiar. Si bien Darío llega "temprano" a Buenos Aires, ya se viene a lo de amigos paraguayos que trabajan en la construcción. Como él comenta, la construcción era lo más fácil para entrar. Al igual que nos señalaron otros entrevistados, "nadie te pide papeles, ni currículum ni nada". "No necesitás nada para empezar a trabajar, ni el primario", nos comentó otro obrero.

Vemos que Darío en 1975 ya tiene conocidos de Humaitá que trabajan en la construcción. Como fuera sugerido por distintos autores ${ }^{25}$, la conformación de la construcción como uno de los ámbitos laborales privilegiados para la inserción de paraguayos se corresponde con procesos sociales por los cuales se comienza a relegar a los migrantes limítrofes a las ocupaciones más marginalizadas $\mathrm{O}$, al menos, a aquellas en las que los nativos empiezan a dejar de desempeñarse hacia mediados de la década de 1970. Estos procesos de segmentación del mercado laboral parecen haberse ido definiendo cada vez más con el correr del tiempo.

\section{Consideraciones finales}

El esfuerzo por contrastar los valores y valoraciones que han rondado a la migración paraguaya hacia Argentina (y hacia el AMBA en particular) sin duda permite poner en cuestión ciertas "cifras imaginarias" 26 por las que en el pasado se ha tendido a sobreestimar la presencia efectiva de migrantes paraguayos en Argentina. Estas cifras fueron muchas veces construidas a partir de percepciones sociales basadas en discursos negativos respecto de la presencia del Otro cultural. Así, la díada conceptual que identifica al paraguayo con las obras en construcción, si bien se apoya en procesos hasta cierto punto "objetivos", ha requerido de una importante cuota de estigmatización y segregación laboral por parte de la sociedad receptora. A través de estos procesos de demarcación, la extracción rural ha sido esgrimida como prueba de una supuesta "inferioridad cultural" de los migrantes, y de una consiguiente "capacidad intrínseca" para realizar tareas de desgaste físico elevado ${ }^{27}$. En este sentido, el origen ruralcampesino de una parte importante de los paraguayos en el AMBA parece haber contribuido a legitimar su inserción laboral en las obras.

Sin embargo, a lo largo de este trabajo, pudimos señalar algunos límites a estas construcciones del "sentido común social". Por un lado, vimos que no es cierto que todos (ni que la mayoría de) los varones paraguayos que migran hacia el AMBA se insertan en la construcción. Lo que sí parece cierto, por el contrario, es que dicha inserción resulta altamente significativa para este grupo,

\footnotetext{
${ }^{25}$ MAGUID, op. cit.; MARSHALL, ORLANSKY, op. cit.

${ }^{26}$ BRUNO, Sebastián. Cifras imaginarias de la inmigración limítrofe en la Argentina.

27 DEL ÁGUILA, op. cit.
} 
dado que casi 4 de cada 10 paraguayos en el AMBA trabajan en una obra ${ }^{28}$. Por otra parte, vimos que muchos de los paraguayos (no todos, ni la mayoría) que trabajan en las obras del AMBA provienen de zonas rurales de Paraguay. Con respecto a esto, al despejar del universo de análisis a aquellos que no poseían trabajo en Paraguay, pudimos notar que los trabajadores provenientes del medio rural alcanzaban el $43 \%$ de quienes actualmente trabajan en la construcción ${ }^{29}$.

Entonces, y más allá de lo limitado y criticable de las miradas que culturizan la capacidad laboral de determinados colectivos sociales, lo aquí analizado nos permitió poner coto a su alcance explicativo real. Sin duda, una vez más, debe concluirse que la explicación que subyace a la presencia significativa de trabajadores paraguayos de origen rural en las obras debe comenzar a buscarse por fuera de los requerimientos técnicos de la producción, para comenzar a buscarse en los procesos sociales más profundos e históricamente construidos de discriminación y segregación socio-laboral de los migrantes.

\section{Bibliografía}

BALÁN, Jorge. Migraciones temporarias y mercado de trabajo rural en América Latina. Buenos Aires: CEDES, 1980.

BALÁN, Jorge. La economía doméstica y las diferencias entre los sexos en las migraciones internacionales: un estudio sobre el caso de los bolivianos en la Argentina. Estudios Migratorios Latinoamericanos, n. 15-16, 1990, 269-294.

BENENCIA, Roberto. Apéndice: La inmigración limítrofe. In DEVOTO, Fernando. Historia de la inmigración en la Argentina. Buenos Aires: Sudamericana, 2003, p. 433-484.

BENENCIA, Roberto; KARASIK, Gabriela. Inmigración limítrofe: los bolivianos en Buenos Aires. Buenos Aires: CEDAL, 1995.

BRUNO, Matías. Las experiencias laborales de paraguayos en Buenos Aires. Un análisis de movilidad ocupacional inicial. Trabajo presentado en: II Taller Paraguay como objeto de estudio de las ciencias sociales, co-organizado por el Grupo de Estudios Sociales sobre Paraguay (UBA) y la Asociación Paraguaya de Estudios de Población. Asunción, 7,8 y 9 de Mayo de 2009.

BRUNO, Sebastián. Cifras imaginarias de la inmigración limítrofe en la Argentina. In NOVICK, Susana (comp.) Migraciones y Mercosur: una relación inconclusa. Buenos Aires: Catálogos, 2010, p. 95-110.

BRUNO, Sebastián. Inserción laboral de los migrantes paraguayos en Buenos Aires. Una revisión de categorías: desde el "nicho laboral" a la "plusvalía étnica". Población y desarrollo, n. 36, 2008, p. 1-17.

\footnotetext{
${ }^{28}$ BRUNO, Movilidad Territorial..., op. cit.

${ }^{29}$ BRUNO, DEL ÁGUILA, op. cit.
} 
BRUNO, Sebastián. Movilidad Territorial y laboral de los migrantes paraguayos en el Gran Buenos Aires. Población y Desarrollo, n. 35, 2008, p. 17-34.

BRUNO, Sebastián; DEL ÁGUILA, Álvaro. Huellas de tierra roja en el cemento porteño. Trabajadores migrantes paraguayos de la construcción en Buenos Aires. Ponencia presentada en: III Taller "Paraguay desde las ciencias sociales". Resistencia, Argentina, 2010.

CACOPARDO, María; LÓPEZ, Silvia. Familia, trabajo y fecundidad de los migrantes de países limítrofes. Estudios Migratorios Latinoamericanos, Año 12, n. 35, 1997, p. 123-145.

CARRÓN, Juan María. Shifting patterns in Migration from bordering countries to Argentina: 1914-1970. International Migration Review, v. 13, n. 3, 1979, p. 475-487.

CERRUTTI, Marcela. Diagnóstico de las poblaciones de inmigrantes en la Argentina. Serie de Documentos de la Dirección Nacional de Población, 2. Buenos Aires: Dirección Nacional de Población, Secretaría del Interior, 2009.

CERRUTTI, Marcela; BRUNO, Matías. La inserción de migrantes paraguayos y peruanos en el mercado de trabajo del Área Metropolitana de Buenos Aires. Estudios Migratorios Latinoamericanos, n. 60, 2006, p. 265-289.

CERRUTTI, Marcela; PARRADO, Emilio. Migración laboral de trabajadores paraguayos a la Argentina: entrada a los mercados de trabajo y trayectorias ocupacionales. Estudios Migratorios Latinoamericanos, n. 48, 2001, p. 369-399.

COURTIS, Corina; PACCECA, María Inés. Género y trayectoria migratoria: mujeres migrantes y trabajo doméstico en el Área Metropolitana de Buenos Aires. Papeles de Población, v. 16, n. 63, 2010, p. 155-185.

CRAGNOLINO, Elsa. Género, trabajo y familia. Trayectorias laborales de mujeres de origen campesino en el norte de Córdoba, Argentina. Estudios del hombre, n. 16, 2003, p. 121-143.

DEL ÁGUILA, Álvaro. A través de la yerba: etnicidad y racionalidad económica entre los trabajadores rurales paraguayos en la industria de la construcción de Buenos Aires. Antípoda, n. 18, 2014, p. 165-187.

DEVOTO, Fernando. Historia de la inmigración en la Argentina. Buenos Aires: Sudamericana, 2003.

FISCHER, Sara; PALAU, Tomás; PEREZ, Noemia. Inmigración y emigración en el Paraguay 1870 - 1960. Documento de trabajo. Asunción: BASE Investigaciones Sociales, 1997.

FLORES COLOMBINO, Andrés. La fuga de los intelectuales. Emigración paraguaya. Montevideo, 1972.

GRIMSON, Alejandro. Fronteras, Estados e identificaciones en el Cono Sur. Cuadernos del IDES, n. 3, 1999, p. 89-103.

GRIMSON, Alejandro. Nuevas xenofobias, nuevas políticas étnicas en Argentina. In GRIMSON, Alejandro; JELIN, Elisabeth (comps.). Migraciones regionales hacia la Argentina. Diferencia, desigualdad y derechos. Buenos Aires: Prometeo, 2006, p. 69-99. 
Indec. Censo nacional de población, hogares y viviendas 2010. Buenos Aires: Instituto Nacional de Estadística y Censos - INDEC, 2012.

Indec. Microdatos por trimestres de la Encuesta Permanente de Hogares. Período 2004-2013. 2014.

MAGUID, Alicia. Migrantes limítrofes en el mercado de trabajo del área metropolitana de Buenos Aires, 1980. Estudios Migratorios Latinoamericanos, n. 18, 1997, p. 147-160.

MARSHALL, Adriana; ORLANSKY, Dora. Inmigración de países limítrofes y demanda de mano de obra en la Argentina, 1940 - 1980. Desarrollo Económico, n. 23, 1983, p. 89-114.

MIRANDA, Ana (comp.). Ahata Che: Juventud, migración y género en el corredor Paraguayo-Argentino. Buenos Aires: FLACSO, 2012.

PASTORE, Carlos. La lucha por la tierra en el Paraguay. Montevideo: Editora Antequera, 1972.

PNUD. Ampliando Horizontes. Emigración Internacional Paraguaya. Disponible en $<$ http://www.py.undp.org/content/paraguay/es/home/library/poverty/ampliandohorizontes--emigracion-internacional-paraguaya.html>. Acceso en: 14.12.2015.

Recibido para publicación en 31.07.2016

Aceptado para publicación en 11.05.2017

Received for publication in July 31, 2016

Accepted for publication in May 11th 2017

ISSN impresso 1980-8585

ISSN eletrônico 2237-9843

http://dx.doi.org/10.1590/1980-85852503880005008 